\title{
Communication
}

\section{Lattice-polarity-driven epitaxy of hexagonal semiconductor nanowires}

Ping Wang, Ying Yuan, Chao Zhao, Xinqiang Wang, Xiantong Zheng, Xin Rong,

Tao Wang, Bowen Sheng, Qingxiao Wang, Yongqiang Zhang, Lifeng Bian, XueLin Yang, Fu-Jun Xu , Zhixin Qin, Xin-Zheng Li, Xixiang Zhang, and Bo Shen

Nano Lett., Just Accepted Manuscript • DOI: 10.1021/acs.nanolett.5b04726 • Publication Date (Web): 22 Dec 2015

Downloaded from http://pubs.acs.org on December 27, 2015

\section{Just Accepted}

"Just Accepted" manuscripts have been peer-reviewed and accepted for publication. They are posted online prior to technical editing, formatting for publication and author proofing. The American Chemical Society provides "Just Accepted" as a free service to the research community to expedite the dissemination of scientific material as soon as possible after acceptance. "Just Accepted" manuscripts appear in full in PDF format accompanied by an HTML abstract. "Just Accepted" manuscripts have been fully peer reviewed, but should not be considered the official version of record. They are accessible to all readers and citable by the Digital Object Identifier (DOI®). "Just Accepted" is an optional service offered to authors. Therefore, the "Just Accepted" Web site may not include all articles that will be published in the journal. After a manuscript is technically edited and formatted, it will be removed from the "Just Accepted" Web site and published as an ASAP article. Note that technical editing may introduce minor changes to the manuscript text and/or graphics which could affect content, and all legal disclaimers and ethical guidelines that apply to the journal pertain. ACS cannot be held responsible for errors or consequences arising from the use of information contained in these "Just Accepted" manuscripts. 


\title{
Lattice-polarity-driven epitaxy of hexagonal semiconductor nanowires
}

\author{
Ping Wang, ${ }^{\dagger}, \xi$ Ying Yuan, ${ }^{\dagger}$, Chao Zhao, ${ }^{\star}$ Xinqiang Wang, ${ }^{*}{ }^{\dagger}$, Xiantong Zheng, ${ }^{\dagger}$ Xin Rong, ${ }^{\dagger}$ Tao \\ Wang, ${ }^{\dagger}$ Bowen Sheng, ${ }^{\dagger}$ Qingxiao Wang, ${ }^{\dagger}$ Yongqiang Zhang, ${ }^{+}$Lifeng Bian, ${ }^{\zeta}$ Xuelin Yang, ${ }^{\dagger}$ Fujun \\ $X u,{ }^{\dagger}$ Zhixin Qin, Xinzheng Li, ${ }^{\dagger}$, Xixiang Zhang, ${ }^{* \dagger}$ and Bo Shen ${ }^{* \dagger, \S}$ \\ ${ }^{\dagger}$ State Key Laboratory of Artificial Microstructure and Mesoscopic Physics, School of Physics, \\ Peking University, Beijing 100871, P. R. China \\ ${ }^{\S}$ Collaborative Innovation Center of Quantum Matter, Beijing 100871, P. R. China \\ ${ }^{\ddagger}$ King Abdullah University of Science and Technology, Division of Physical Science and \\ Engineering and Core Labs, Thuwal 23955-6900, Kingdom of Saudi Arabia \\ ${ }^{\zeta}$ Suzhou Institute of Nano-Tech and Nano-Bionics, Chinese Academy of Science, Suzhou \\ 215123, P. R. China
}
KEYWORDS: Nanowires, indium nitride, lattice-polarity, molecular beam epitaxy, hexagonal semiconductor

\begin{abstract}
Lattice-polarity-driven epitaxy of hexagonal semiconductor nanowires (NWs) is demonstrated on InN NWs. In-polarity InN NWs form typical hexagonal structure with pyramidal growth front, whereas N-polarity InN NWs slowly turn to the shape of hexagonal
\end{abstract}


pyramid and then convert to an inverted pyramid growth, forming diagonal pyramids with flat surfaces and finally coalescence with each other. This contrary growth behavior driven by lattice-polarity is most likely due to the relatively lower growth rate of the $(000 \overline{1})$ plane, which results from the fact that the diffusion barriers of In and $\mathrm{N}$ adatoms on the $(0001)$ plane $(0.18$ and $1.0 \mathrm{eV}$, respectively) are about two-fold larger in magnitude than those on the $(000 \overline{1})$ plane $(0.07$ and $0.52 \mathrm{eV}$ ), as calculated by first-principles density functional theory (DFT). The formation of diagonal pyramids for the N-polarity hexagonal NWs affords a novel way to locate quantum dot in the kink position, suggesting a new recipe for the fabrication of dot-based devices.

Semiconductor nanowires (NWs), such as $\mathrm{GaN}, \mathrm{ZnO}, \mathrm{CdS}$, InAs, etc, have attracted much attention during the past years due to their nearly prefect crystalline quality and a wide range of applications including highly efficient, nanoscale optoelectronic and electronic devices. ${ }^{1-5}$ To achieve such high quality semiconductor NWs, one-dimensional (1D) growth is essential. Most of the semiconductors NWs, such as GaN, ZnO and CdS with well-manipulated 1D growth, are in hexagonal phase in which the NWs easily grow along c-direction. The shape and diameter of such NWs are therefore well-controlled. Recently, a lattice-polarity effect was observed in some NWs, due to the partial ionicity of the chemical bonds, which may lead to different growth behaviors of these nanowires and the final orientation to be different. ${ }^{6-10}$ Based on this polarity effect, new nanostructures such as kinks and tripods have been reported, especially in the transition region between cubic and hexagonal phases. ${ }^{11-13}$ Even though these findings are exciting, the mechanism underlying the formation of these different structures and their correlation with the polarity effect remain unclear. Moreover, most of these structures are based on $\mathrm{GaN}$ or II-VI semiconductors $(\mathrm{ZnO}, \mathrm{ZnS}, \mathrm{CdS}$, etc) with relatively large bandgaps and low electron mobility, ${ }^{1,3,5}$ which limits their application in solar-related, ultrafast electronic, and other 
optoelectronic devices. Similar structures in semiconductors with small bandgaps and high electron mobility, ideally InN, are highly desired. ${ }^{14}$

In spite of the seemingly clear desire for the growth of polarity-induced InN nanowires, reports on such studies are rare, mainly due to fact that the low maximum growth temperature and the lack of suitable substrate. ${ }^{15} \mathrm{InN}$ is a member of III-nitrides with a narrow direct band gap of $0.64 \mathrm{eV}$ at room temperature, high electron mobility $\left(4400 \mathrm{~cm}^{2} / \mathrm{Vs}\right.$ at $\left.300 \mathrm{~K}\right)$, small effective mass $\left(0.07 \mathrm{~m}_{0}\right)$, and high saturation velocity $\left(2.5 \times 10^{17} \mathrm{~cm} / \mathrm{s}\right) .{ }^{14-18} \mathrm{InN} \mathrm{NWs}$ have thus emerged as an excellent candidate for several nanoscale optoelectronic applications, such as nano-LEDs, ${ }^{19,20}$ nano-lasers, ${ }^{1,21}$ nano-solar cells, ${ }^{22}$ nano-biochemistry sensors, ${ }^{23}$ and single photon emitters, ${ }^{24-26}$ etc. In addition, the tunable bandgap energy of $\operatorname{InGaN}(0.64-3.43 \mathrm{eV})$ matches perfectly with the solar spectrum. Because of a recently discovered light-concentrating property of standing nanowires, ${ }^{27,28}$ InGaN provides a promising approach to enhancing the efficiency of nano-solar cells. Here, we explore the lattice-polarity-driven epitaxy mechanism using InN.

Our experiments clearly reveal that lattice-polarity extensively influences the growth behavior of InN NWs, as was reported earlier for GaN and II-VI semiconductors. Besides this and more interestingly, the InN NWs exhibit completely different features in a well-controlled manner from the ones reported. In-polarity NWs form typical hexagonal structure with pyramidal growth front, whereas the N-polarity NWs slowly turn to the shape of hexagonal pyramid and then convert into inverted pyramid growth, forming diagonal pyramids with flat surfaces, which finally coalescence with each other. A quantum dot can easily form at the kink position. This contrary growth behavior can be rationalized using kinetic Wulff's plot combined with firstprinciples density functional theory. Since the kink position is well-connected with the structures on both sides, especially with the diagonal pyramids with flat surfaces on the top, fabrication of 
devices becomes convenient. The structure we observed thus suggests a new recipe for the fabrication of dot-based devices, which could be applicable to a wide range of nanoscale optoelectronic and solar systems.

An observation of an unusual nanowire, as shown in Figure 1, led us to be interested in the lattice-polarity-driven epitaxy of InN NWs. These InN NWs were grown by SVTA radiofrequency plasma-assisted molecular beam epitaxy (RF PA-MBE) on pillar-patterned GaN templates, which were fabricated by nanoimprint technology, without using any foreign mask or catalyst. N-rich growth conditions were maintained with a nitrogen flow rate of $\sim 1.2$ standard cubic centimeters per minute (sccm), RF plasma forward power of $400 \mathrm{~W}$, and In beam flux of $\sim 2.0 \times 10^{-7}$ Torr. The corresponding V/III ratio was about 5 . Most of the InN NWs exhibited regular shape with hexagonal pyramidal front, with nearly uniform diameter of $\sim 400 \mathrm{~nm}$ and six smooth sidewalls. This morphology is similar to other hexagonal semiconductor NWs, such as GaN, CdS, and ZnO. ${ }^{1,3,7,29}$ The most interesting observation is that a few nanowires ending with very big, inverted pyramid-shaped, hexagonal cap with diameter of $3 \mu \mathrm{m}$ co-existed with the much smaller wires as shown in Figure 1a. The tilt-view scanning electron microscopy (SEM) image in Figure $1 \mathrm{~b}$ shows that the InN NW changed its growth behavior at a kink position and then an inverted hexagonal pyramid with a flat top surface grew.

To understand the mechanism behind, Titan-CsP probe-corrected scanning transmission electron microscopy (STEM) was performed on the sample and the corresponding TEM images are shown in Figure 2a. Although the inverted pyramid was slightly separated from the bottom wire during the focus ion beam (FIB) preparation of the TEM specimen, GaN pillars at bottom and $\mathrm{InN}$ wires can clearly be distinguished. High resolution STEM was performed at different positions (marked in Figure 2a as b, $\mathrm{c}$ and d), which corresponded to the GaN pillar, InN wire 
and InN inverted pyramid, respectively. Corresponding annular bright field (ABF) STEM images at atomic level are shown in Figure 2b, 2c and 2d. In the ABF-STEM images, the darkest spots correspond to the heaviest atoms. ${ }^{30}$ As such, we are able to configure the atomic structure, which is shown in the ABF-STEM images in which the blue and red balls represent the Ga and In atoms, respectively, while the gray ones indicate the $\mathrm{N}$ atoms. By comparing the experimental atomic structures with the schematic ones shown in Figure 2e, we determined that the latticepolarity of the GaN pillar, InN wire and inverted pyramid is Ga-, In- and N-polarity, respectively. In addition, we also measured other $\mathrm{InN}$ NWs that have the conventional shape without the inverted pyramid and the STEM results showed that they are all In-polarity. Furthermore, the lattice-polarity was actually also confirmed by chemical wet etching, which showed the same result as the STEM measurement (data not shown here).

Based on above results, the lattice-polarity seems to be the origin for the formation of the inverted InN pyramid. To further clarify and demonstrate this point, systematical study on the lattice-polarity-driven growth of $\mathrm{InN} \mathrm{NWs}$ has been performed on pillar-patterned $\mathrm{GaN}$ templates with different polarities. The growth conditions for In- and N-polarity InN NWs were the same except that the N-polar NWs were grown at $600^{\circ} \mathrm{C}$, which was about $100{ }^{\circ} \mathrm{C}$ higher than that of In-polarity NWs. The lattice-polarity of the InN NWs was controlled by the pillarpatterned GaN templates; in other words, the $\mathrm{Ga}(\mathrm{N})$-polar pillar leads to $\mathrm{In}(\mathrm{N})$-polarity NWs. The entire growth procedure was monitored in situ by reflection high energy electron diffraction (RHEED) and the RHEED patterns at different stages are shown in Figure 3. Before the deposition of InN, streaky patterns along the [11 $\overline{2} 0]$ and [1 $\overline{1} 00]$ azimuths are clearly observed, as shown in Figure 3 ( $0 \mathrm{~min})$, indicating that the surfaces for Ga- and N-polarity pillar-patterned GaN templates were flat. Thereafter, the changes in the RHEED patterns over time differ with 
opposite lattice polarities. The most obvious feature is that along with the growth time, the RHEED pattern of the In-polarity InN NWs remains spotty, whereas for the N-polarity NWs, the pattern changes from spotty to streaky, suggesting quite different growth behaviors.

In the case of In-polarity, the RHEED pattern along the [11 20$]$ azimuth gradually changes from streaky to slightly spotty with weakly forked tails (as shown in Figure 3a (10 min)) at the initial growth stage, indicating that the surface becomes rough. The pattern then becomes brightly spotty with well-defined crossed-chevrons that barely budged in the following process (Figure 3a (30 min)). However, the RHEED pattern along the [1100] azimuth remains mostly unchanged, with the chevrons observed during the full process. The observation of the offnormal streaks being elongated from the center spot pattern indicates that the top surface has a faceted structure. ${ }^{31,32}$ As such, it is not surprising that chevron patterns indicate that the NWs have a pyramid-shaped top surface. The interesting feature is that the chevrons along the [11 $\overline{2} 0]$ azimuth form an included angle of $64^{\circ}$ (Figure 3a $(30 \mathrm{~min})$ ), which indicates that the top facets of the NWs are of the $\{1 \overline{1} 03\}$ family. This is similar to Ga-polarity GaN NWs that have a pyramidal top with $\{1 \overline{1} 0 \mathrm{n}\}$ planes. $^{33,34}$

In the case of N-polarity, as shown in Figure 3c and 3d, the RHEED patterns gradually change from streaky to chevrons with a center streaky line, indicating that the NWs have faceted sidewalls with flat top surface. Then, the patterns return to being streaky and remain so until the end of the growth, suggesting a top flat surface with no sidewall. This novel observation is unexpected, since it suggests that the tilted top facets were gradually annihilated and merged together. Such an observation has not been previously reported and is quite difficult to understand at such extremely N-rich growth condition for nanowires. 
Above interpretation of the RHEED patterns is supported by the direct observations of the morphology of NWs, as shown in Figure 4. The In-polar InN NWs exhibit regular shape with hexagonal pyramid-shaped front, nearly uniform diameter and six smooth sidewalls (Figure 4a). This morphology appear to be almost the same as previously reported hexagonal wires and sidewalls of $\{1 \overline{1} 00\}$ m-faces. ${ }^{17,20,22,25,33}$ It is quite interesting that the N-polar InN NWs become narrower and narrower to form a kink and suddenly begin to broaden (Figure 4b). Finally, the NWs coalesce with each other and form a flat surface, with an inverted pyramid shape from the kink, which is the same as that shown in Figure 1. The top-view SEM image in the inset also clearly shows the aggregation of the NWs.

To further explicate the growth behaviors of InN NWs, we present in Figure 5a a schematic of the process with four growth stages corresponding to the RHEED patterns shown in Figure 3. Meanwhile, the growth process of opposite polarities nanowires also divided into four stages (Figure 5b and 5c), which tally well with the schematic model.

It is easy to understand that the growth mechanism of In-polar NWs is almost the same as that of hexagonal semiconductor wires such as $\mathrm{ZnO}, \mathrm{CdS}$, and $\mathrm{GaN} .^{7,35,36}$ However, the growth behaviors of N-polar InN NWs are difficult to understand, particularly the inverted pyramid growth. It is possible to understand that the top face of the N-polar NWs almost always keep flat whereas the In-polar ones keep needle-like. This is most likely due to the anisotropic growth velocities along different orientations, which can be described by the kinetic Wulff's plots ( $v$ plot). ${ }^{36,37,38}$ Given the features of In- and N-polar InN NWs, it is convenient to deduce the relative growth-rate ratios of the $(0001),(1 \overline{1} 03),(1 \overline{1} 00)$, and $(000 \overline{1})$ planes to be 5.3:1.2:1.8:1 based on the data in Figures $5 \mathrm{~b}$ and $5 \mathrm{c}$, which are the key points of the $v$-plot. (These growth rates ratios are not unique, but change with the growth conditions, including the growth 
temperature and the V/III ratio.) To complete the $v$-plot, the growth rates between these special orientations were extrapolated as long as they did not modify the shape of the $v$-plot. ${ }^{36,39}$ The calculated schematic $v$-plot of $\mathrm{InN}$ is shown in Figure 6a. It is shown that the growth rate of the InN (0001) plane is significantly larger than that of the $(000 \overline{1})$ plane, and thus we are able to predict that the In-polar NWs have a pyramid growth front with $\{1 \overline{1} 0 \mathrm{n}\}$ planes while the N-polar NWs keep flat top, as schematized in the bottom of Figure 6a.

To understand the physics behind the difference in growth rates, a detailed analysis of the diffusion barriers of In and $\mathrm{N}$ adatoms on InN surfaces have been performed by first-principles density functional theory calculations as implemented in the Vienna Ab-Initio Simulation Package (VASP). In the calculation, we used the Perdew-Burke-Ernzerhof (PBE) version of the generalized gradient approximation $(\mathrm{GGA})$ and the projector-augmented wave method. ${ }^{40,41} \mathrm{~A}$ plane-wave cutoff energy of $550 \mathrm{eV}$ was used and a $4 \times 4 \times 1 \mathrm{k}$-point mesh was set. To calculate the diffusion barriers on the thermodynamically stable sites, we used two kinds of atomic structures that both terminated with In atoms, ${ }^{42,43}$ as shown in Figure $6 \mathrm{~b}$. The $(2 \times 2)$ supercells used for the calculations contained six bilayers with $\mathrm{a} \sim 16 \AA$ thick vacuum: the bottom three bilayers were fixed in the bulk configurations, but the upper three bilayers and the adatoms were allowed to relax. The diffusion barriers were calculated using the climbing image nudged elastic band (CI-NEB) method ${ }^{44,45}$ in which all the force components that were perpendicular to the tangent of the reaction pathway were reduced to be less than $0.05 \mathrm{eV} / \AA \AA$. As shown in Figure 6c, the diffusion barriers of $\mathrm{In}$ and $\mathrm{N}$ adatoms on the (0001) plane (i.e., In-polarity) are $0.18 \mathrm{eV}$ and $1.00 \mathrm{eV}$, respectively, which are about 2 -fold in magnitude larger than $0.07 \mathrm{eV}$ and $0.52 \mathrm{eV}$ on

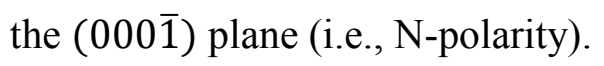


It is well known that the diffusion length of adatoms is proportional to $\exp \left[-E_{A} /\left(2 k_{B} T\right)\right]$, where $E_{A}, k_{B}, T$ are the diffusion barrier, Boltzmann constant, and growth temperature, respectively. ${ }^{46}$ Therefore, the diffusion lengths of In and N adatoms on the (0001) plane are larger than those on the (0001) plane. The former is probably even much larger than the estimated one, since the growth temperature of N-polar InN NWs was $100{ }^{\circ} \mathrm{C}$ higher than that of the In-polar InN NWs. ${ }^{47}$ This difference is probably the essential factor in the anisotropic growth velocity, as shown in Figure 6a. Compared with the In-polarity case, the relatively large diffusion lengths in the Npolarity case make it possible for adatoms to migrate to steps, kinks, and vacancies that are propitious to maintaining two-dimensional (2D) growth and that result in a lower growth rate under the same growth conditions.

Based on the $v$-plot and calculation results, we propose a lattice-polarity-driven growth mechanism to explain the intriguing phenomenon observed in InN NWs. At the initial stage, both In- and N-polar InN NWs show 3D growth, which is an inevitable product of N-rich growth conditions and the release of stress that results from the $10.8 \%$ lattice mismatch between InN and $\mathrm{GaN}^{48}$

I. In-polar InN NWs: The primary 3D growth and the large growth rate of the (0001) plane cause the c-plane to be rapidly annihilated to form a pyramid-shaped growth front that is surrounded by facets with relatively low growth rates, i.e., $\{1 \overline{1} 00\}$ and $\{1 \overline{1} 0 n\}$ (in our case $n=3$ ) planes. This stabilized shape will be maintained in the subsequent growth process except that the diameter is slightly broadened due to the lateral growth as shown in Figure $5 \mathrm{~b}$ (at 60, 90, and 150 $\min )$.

II. N-polar InN NWs: The incipient 3D growth causes the nanowires to exhibit a prismoid shape with a flat top as illustrated in Figure $5 \mathrm{c}(10 \mathrm{~min})$, which results from the lowest growth 
rate of the $(000 \overline{1})$ plane. In this process, the prismoid shape gradually shrinks until the full relaxation of the lattice strain and then converts into a conical or near-conical (with a small flat top) shape, as shown in Figure $5 \mathrm{c}(30 \mathrm{~min})$. However, according to the $v$-plot, $\operatorname{InN}$ nanostructures should be surrounded by facets with low growth rates for N-polarity, i.e., the $(000 \overline{1})$ and $\{1 \overline{1} 00\}$ planes. Obviously, the conical or near-conical shape is not stable. Finally, the $(000 \overline{1})$ plane appears again and expands gradually. With expansion of the $(000 \overline{1})$ plane, more In atoms are directly adsorped on the top rather than on the bottom or diffused to other nanowires, leading to stronger lateral growth and diagonal pyramids (Figure $5 \mathrm{c}(50 \mathrm{~min}))$. That is also the reason for the lack of $\{1 \overline{1} 00\}$ planes, which is not contrary to the kinetic Wulff's plot.

From the above analysis, there is no doubt that the N-polarity leads InN NWs to experience inverted-pyramid growth, which explains our observations in Figure 1. Although it is extremely difficult to show the precise atomic structure of the inversion point for lattice polarity, which is too narrow and exhibits too much strain to be analyzed in TEM measurements, we can still reasonably assume that inversion of the lattice-polarity at the kink is most likely due to the formation of stacking faults, which are often observed in such NWs. ${ }^{11,33,49}$ Once the lattice polarity converts from In- to N-polarity, it will lead to inverted pyramid growth since the bottom In-polar NWs are almost free from strain.

Interestingly, the lattice-polarity-driven growth behavior reported here for InN NWs is not limited to InN itself. It should be applicable to other hexagonal semiconductor nanowires, such as $\mathrm{GaN}, \mathrm{ZnO}, \mathrm{CdS}$ and so on.

In conclusion, we have confirmed that opposite lattice polarity leads to different growth behaviors in InN NWs. Elongated NWs with typical pyramid-shaped growth fronts were observed for In-polarity. On the other hand, a hexagonal pyramid was gradually formed for the 
N-polarity NWs, which was followed by inverted pyramid-shaped growth, forming diagonal pyramids. The inverted pyramids exhibit flat top surfaces and broadened diameters during growth and finally coalescence with each other. This lattice-polarity-driven growth behavior is most likely due to the fact that the diffusion barriers for In and $\mathrm{N}$ adatoms on the (0001) plane are about two-fold larger in magnitude than those on the $(000 \overline{1})$ plane, as predicted from DFT calculations, that result in anisotropic growth velocities. A semi-quantitative lattice-polaritydriven growth mechanism was proposed to explain the diametrically opposed growth behaviors. We suggest that the formation of diagonal pyramids for the N-polarity NWs may provide a novel way to locate quantum dot in the kink position and thus improve the fabrication of dot-based optoelectronic devices since they finally coalescence with each other. 
Figures
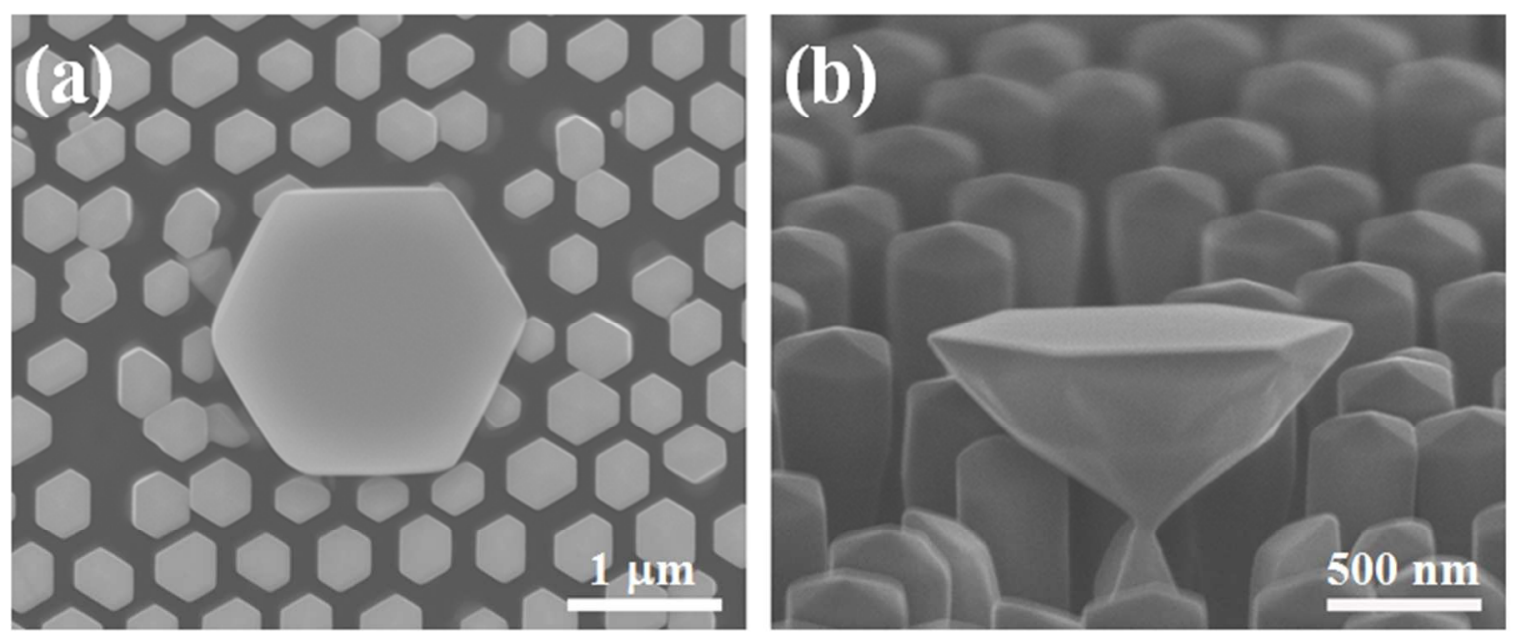

Figure 1. (a) Top-view and (b) tilt-view SEM images of the inverted pyramid that emerged in In-polarity InN NWs.

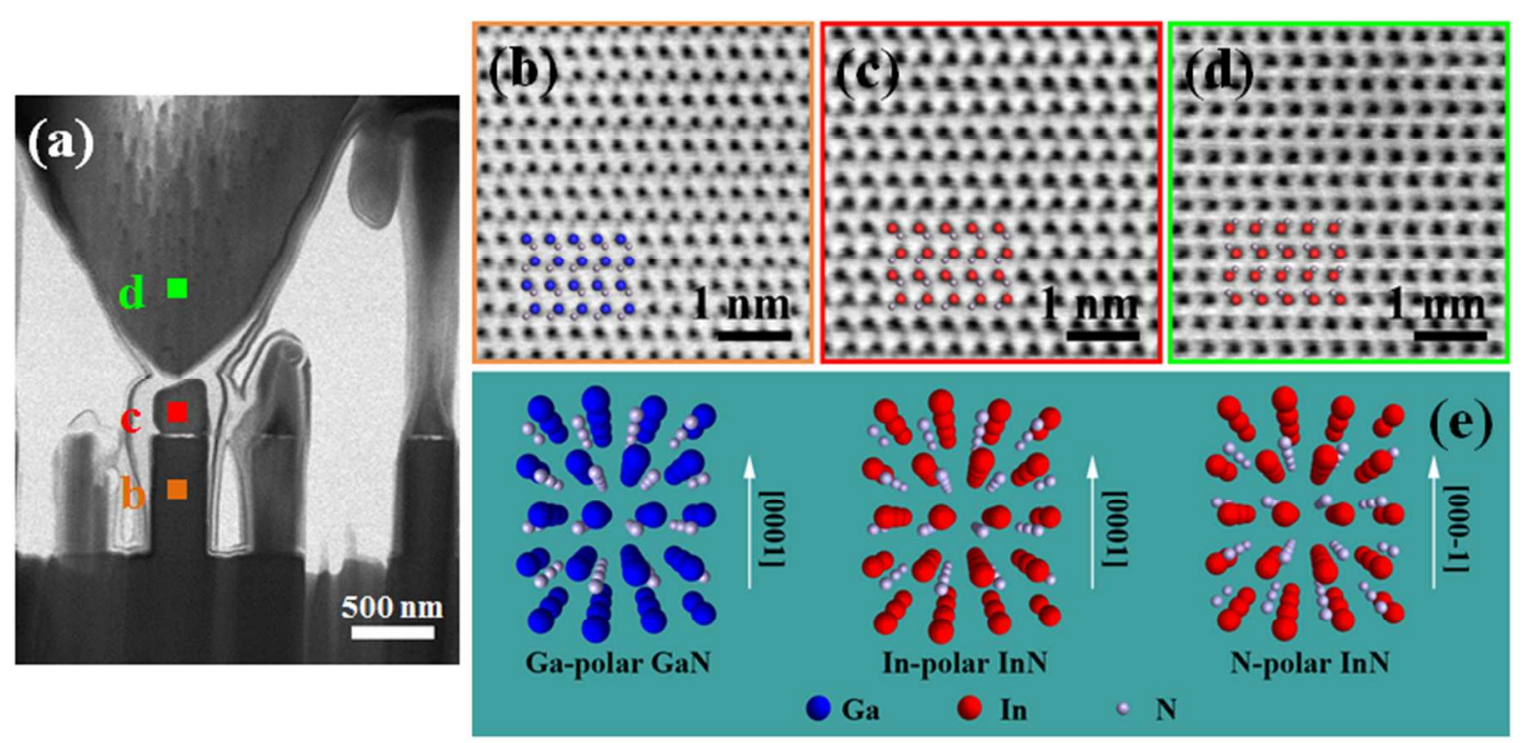

Figure 2. (a) TEM image of an inverted InN NW. (b), (c), and (d) are ABF-STEM images of the zones marked as brown square ( $\mathrm{GaN}$ pillar), red square ( $\mathrm{InN}$ wire), and green square (inverted pyramid) in (a), respectively. Ga (blue balls), In (red balls), and $\mathrm{N}$ (gray balls) atoms were 
embedded in the ABF-STEM images to show the atomic stack order. (e) Atomic schematics of Ga-polarity GaN, In- and N-polarity InN.

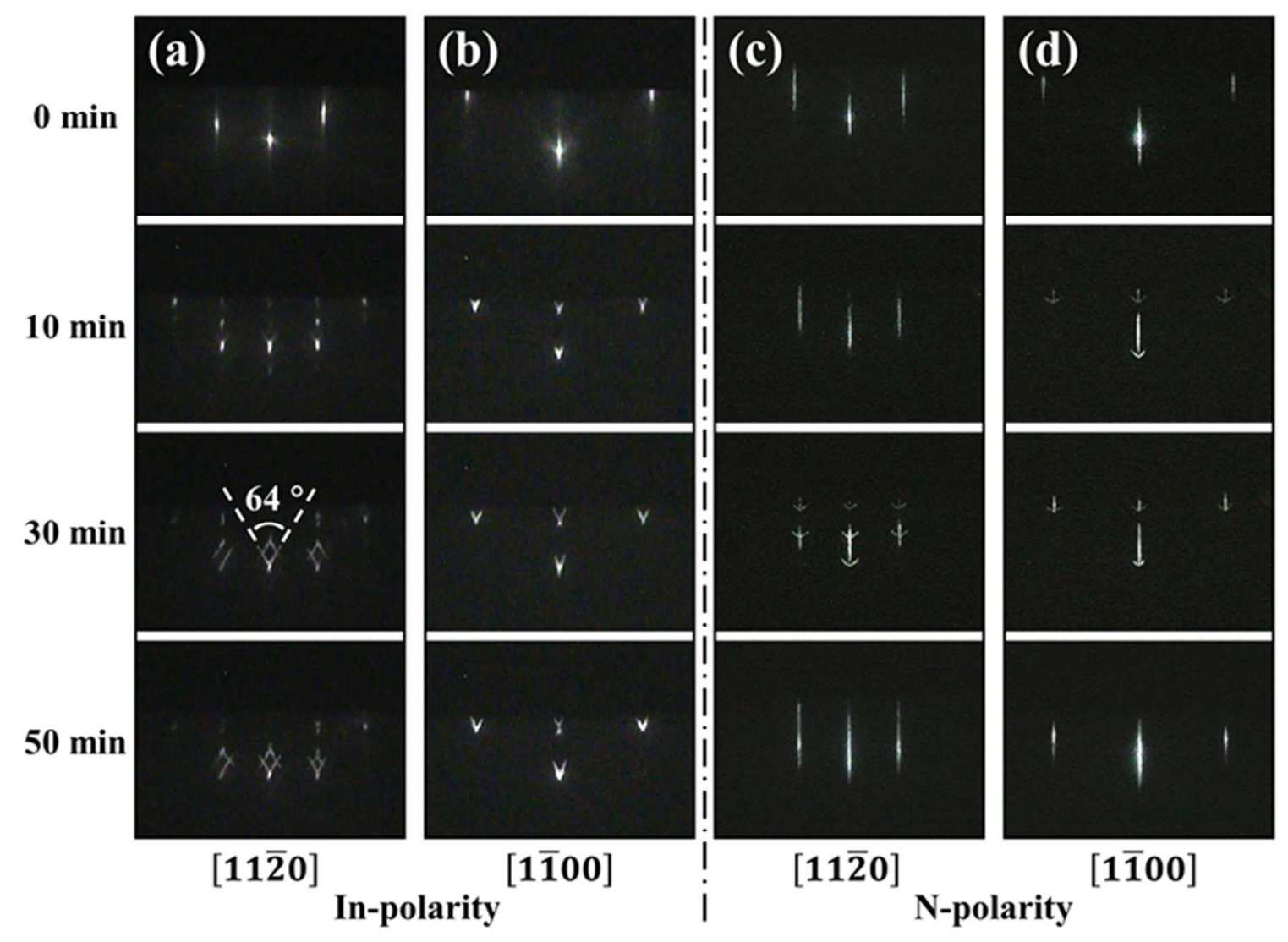

Figure 3. Evolution of RHEED patterns of (a, b) In- and (c, d) N-polarity InN NWs along the $[11 \overline{2} 0]$ and $[1 \overline{1} 00]$ azimuths with growth time of $\mathrm{t}=0,5,30$, and $60 \mathrm{~min}$. The white dashed line in (a) shows an included angle of $64^{\circ}$ that indicates a diffraction pattern from the $\operatorname{InN}\{1 \overline{1} 03\}$ family. 

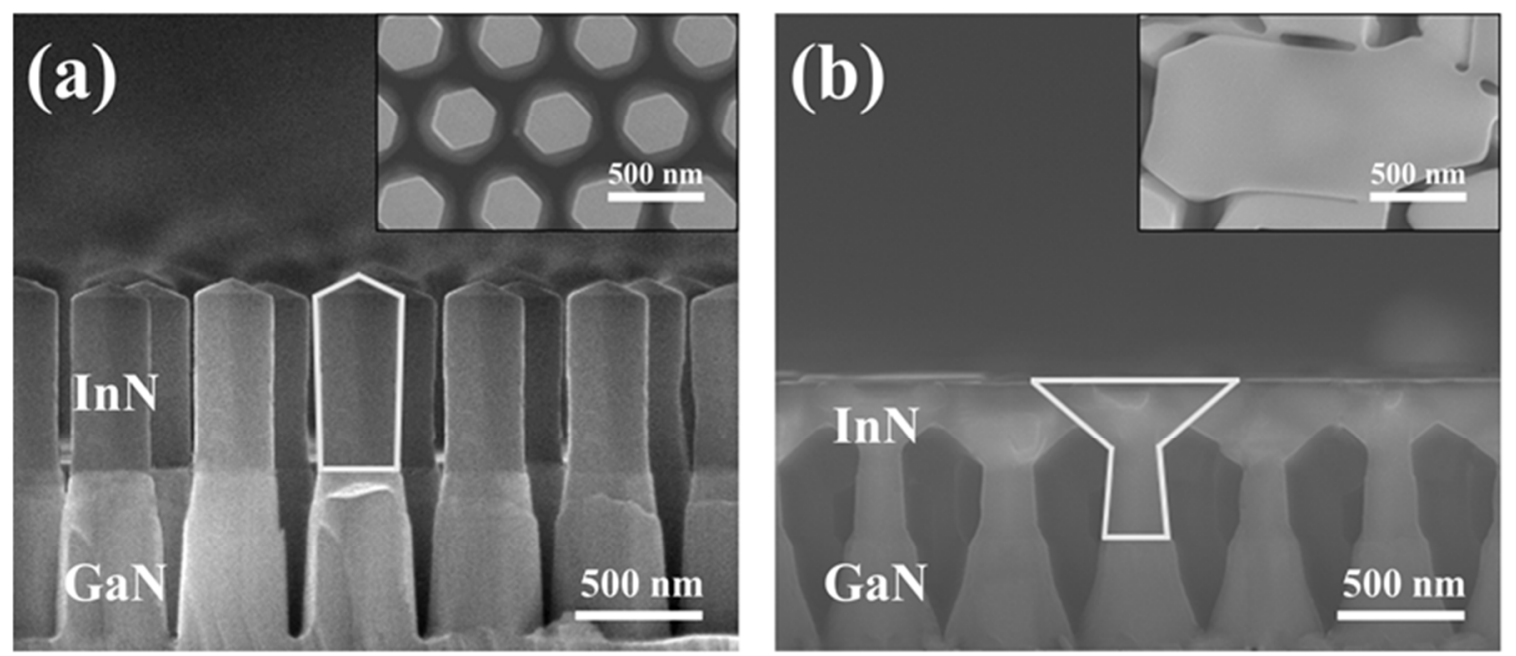

Figure 4. Cross-section SEM images of (a) In-polarity and (b) N-polarity InN NWs. Insets are top-views of the corresponding nanowires. The gray frames show the outline of opposite polarities InN NWs with pyramid-shaped growth fronts and inverted growth fronts, respectively.
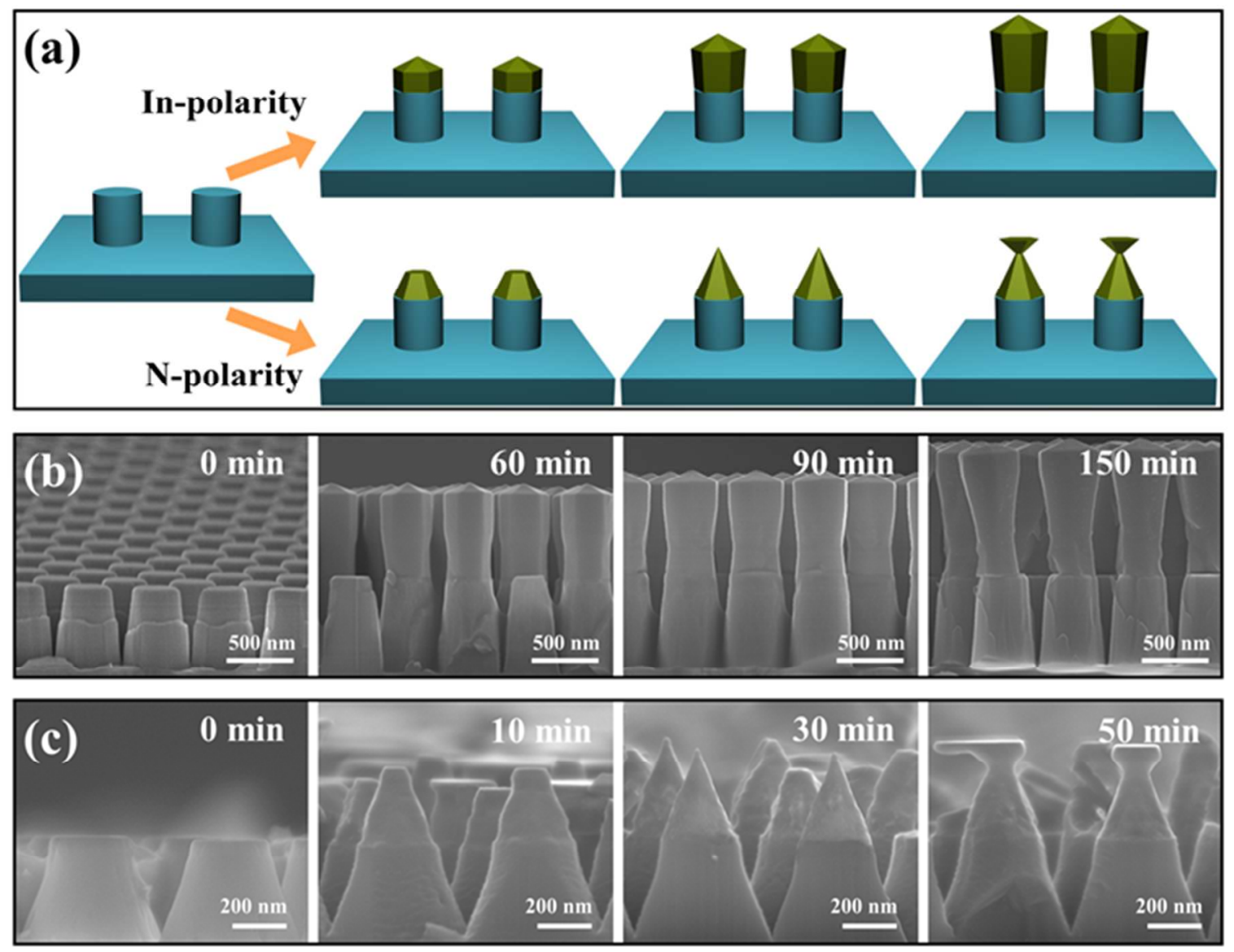
Figure 5. (a) A schematic model of different growth stages of InN NWs with opposite polarities. Tilt-view SEM images of (b) In- and (c) N-polarity InN NWs with growth time.
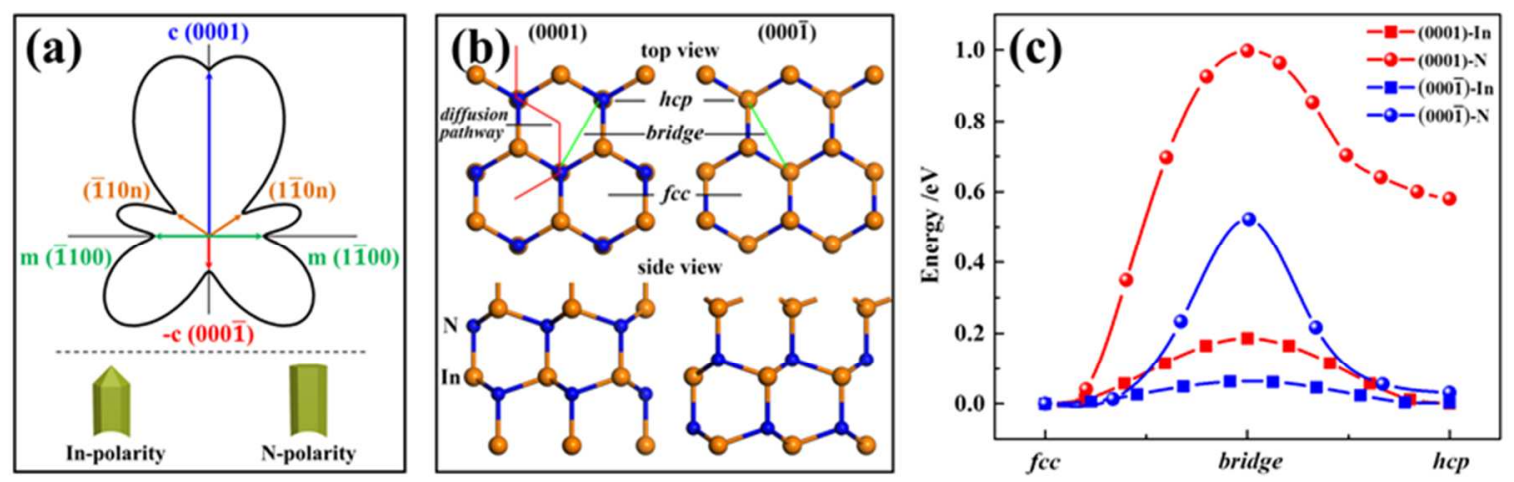

Figure 6. (a) The kinetic Wulff's plots for InN across the (1120) plane: the blue, brown, green, and red arrows correspond to the $(0001),\{1 \overline{1} 0 \mathrm{n}\},\{1 \overline{1} 00\}$, and $(000 \overline{1})$ planes, and solid line shows the extrapolated velocity values. (b) Atomic structures (top view and side view) for (0001) and $(000 \overline{1})$ In terminated surfaces. The red line at the top left marks the diffusion pathway corresponding to (c). Three key adsorption sites, fcc, bridge, and hcp, are also marked in this figure. (c) Total energy for In and $\mathrm{N}$ adatoms on (0001) and (0001) surfaces, which was calculated based on the atomic structure shown in (b). Zero energy corresponds to the energetically lowest adsorption site. 


\section{Corresponding Authors}

*E-mail: wangshi@pku.edu.cn; xixiang.zhang@kaust.edu.sa; bshen@pku.edu.cn.

\section{Funding sources}

National Basic Research Program of China (No. 2012CB619300 and 2013CB632800);

National Natural Science Foundation of China (No. 61225019, 61521004 and 61376060);

Open Fund of the State Key Laboratory on Integrated Optoelectronics and King Abdullah University of Science and Technology;

Open-Lab program (Project No. 12ZS01) of the Key Laboratory of Nanodevices and Applications, Suzhou Institute of Nano-Tech and Nano-Bionics (SINANO), Chinese Academy of Sciences (CAS).

\section{Notes}

The authors declare no competing financial interest.

\section{Acknowledgment}

This work was partly supported by the National Basic Research Program of China (No. 2012CB619300 and 2013CB632800), the National Natural Science Foundation of China (No. 61225019, 61521004 and 61376060), the Open Fund of the State Key Laboratory on Integrated Optoelectronics and King Abdullah University of Science and Technology, and the Open-Lab program (Project No. 12ZS01) of the Key Laboratory of Nanodevices and Applications, Suzhou Institute of Nano-Tech and Nano-Bionics (SINANO), Chinese Academy of Sciences (CAS). The authors are grateful to Prof. Weikun Ge for his critical reading of the manuscript. We also acknowledge Dr. Kun Li for his support in the TEM experiments. 


\section{References}

(1) Lu, Y. J.; Kim, J.; Chen, H. Y.; Wu, C.; Dabidian, N.; Sanders, C. E.; Wang, C. Y.; Lu, M. Y.; Li, B. H.; Qiu, X.; Chang, W. H.; Chen, L. J.; Shvets, G.; Shih, C. K.; Gwo, S. Science 2012, $337,450-3$.

(2) Wang, Z. L.; Song, J. Science 2006, 312, 242.

(3) Oulton, R. F.; Sorger, V. J.; Zentgraf, T.; Ma, R. M.; Gladden, C.; Dai, L.; Bartal, G.; Zhang, X. Nature 2009, 461, 629-32.

(4) Wallentin, J.; Anttu, N.; Asoli, D.; Huffman, M.; Aberg, I.; Magnusson, M. H.; Siefer, G.; Fuss-Kailuweit, P.; Dimroth, F.; Witzigmann, B.; Xu, H. Q.; Samuelson, L.; Deppert, K.; Borgstrom, M. T. Science 2013, 339, 1057-60.

(5) Yan, R.; Gargas, D.; Yang, P. Nature Photon. 2009, 3, 569-576.

(6) Yang, R.; Ding, Y.; Wang, Z. L. Nano Lett. 2004, 4, 1309.

(7) Lee, S. H.; Minegishi, T.; Park, J. S.; Park, S. H.; Ha, J.-S.; Lee, H. J.; Lee, H. J.; Ahn, S.; Kim, J.; Jeon, H.; Yao, T. Nano Lett. 2008, 8, 2419.

(8) Kim, K. S.; Jeong, H.; Jeong, M. S.; Jung, G. Y. Adv. Funct. Mater. 2010, 20, 3055-3063.

(9) Hestroffer, K.; Leclere, C.; Bougerol, C.; Renevier, H.; Daudin, B. Phys. Rev. B 2011, 84, 245302 .

(10) Fernandez-Garrido, S.; Kong, X.; Gotschke, T.; Calarco, R.; Geelhaar, L.; Trampert, A.; Brandt, O. Nano Lett. 2012, 12, 6119-25. 
(11) Yan, L.; Jahangir, S.; Wight, S. A.; Nikoobakht, B.; Bhattacharya, P.; Millunchick, J. M. Nano Lett. 2015, 15, 1535-9.

(12) Utama, M. I. B.; Zhang, Q.; Jia, S.; Li, D.; Wang, J.; Xiong, Q. ACS Nano 2012, 6, 2281.

(13) Schuster, F.; Hetzl, M.; Weiszer, S.; Garrido, J. A.; de la Mata, M.; Magen, C.; Arbiol, J.; Stutzmann, M. Nano Lett. 2015, 15, 1773-9.

(14) Chang, Y. L.; Mi, Z.; Li, F. Adv. Funct. Mater. 2010, 20, 4146.

(15) Wang, X.; Liu, S.; Ma, N.; Feng, L.; Chen, G.; Xu, F.; Tang, N.; Huang, S.; Chen, K. J.; Zhou, S.; Shen, B. Appl. Phys. Express 2012, 5, 015502.

(16) Wu, J.; Walukiewicz, W.; Yu, K. M.; Ager, J. W.; Haller, E. E.; Lu, H.; Schaff, W. J.; Saito, Y.; Nanishi, Y. Appl. Phys. Lett. 2002, 80, 3967.

(17) Hu, M. S.; Wang, W. M.; Chen, T. T.; Hong, L. S.; Chen, C. W.; Chen, C. C.; Chen, Y. F.; Chen, K. H.; Chen, L. C. Adv. Funct. Mater. 2006, 16, 537-541.

(18) Grandal, J.; Sánchez-García, M. A.; Calleja, E.; Luna, E.; Trampert, A. Appl. Phys. Lett. 2007, 91, 021902.

(19) Lin, H. W.; Lu, Y. J.; Chen, H. Y.; Lee, H. M.; Gwo, S. Appl. Phys. Lett. 2010, 97, 073101.

(20) Sekiguchi, H.; Kishino, K.; Kikuchi, A. Appl. Phys. Lett. 2010, 96, 231104.

(21) Wu, C. Y.; Kuo, C. T.; Wang, C. Y.; He, C. L.; Lin, M. H.; Ahn, H.; Gwo, S. Nano Lett. 2011, $11,4256-60$. 
(22) Wierer Jr, J. J.; Li, Q.; Koleske, D. D.; Lee, S. R.; Wang, G. T. Nanotechnology 2012, 23, 194007.

(23) Lim, W.; Wright, J. S.; Gila, B. P.; Pearton, S. J.; Ren, F.; Lai, W. T.; Chen, L. C.; Hu, M. S.; Chen, K. H. Appl. Phys. Lett. 2008, 93, 202109.

(24) Deshpande, S.; Heo, J.; Das, A.; Bhattacharya, P. Nature Commun. 2013, 4, 1675.

(25) Kim, J. H.; Ko, Y. H.; Gong, S. H.; Ko, S. M.; Cho, Y. H. Sci. Rep. 2013, 3, 2150.

(26) Holmes, M. J.; Choi, K.; Kako, S.; Arita, M.; Arakawa, Y. Nano Lett. 2014, 14, 982-6.

(27) Krogstrup, P.; Jørgensen, H. I.; Heiss, M.; Demichel, O.; Holm, J. V.; Aagesen, M.; Nygard, J.; Fontcuberta i Morral, A. Nature Photon. 2013, 7, 306-310.

(28) Ko, W. S.; Tran, T. T.; Bhattacharya, I.; Ng, K. W.; Sun, H.; Chang-Hasnain, C. Nano Lett. 2015, 15, $4961-7$.

(29) Liao, C. H.; Chang, W. M.; Chen, H. S.; Chen, C. Y.; Yao, Y. F.; Chen, H. T.; Su, C. Y.; Ting, S. Y.; Kiang, Y. W.; Yang, C. C. Opt. Express 2012, 20, 15859.

(30) De la Mata, M.; Magen, C.; Gazquez, J.; Utama, M. I. B.; Heiss, M.; Lopatin, S.; Furtmayr, F.; Fernández-Rojas, C. J.; Peng, B.; Morante, J. R.; Rurali, R.; Eickhoff, M.; Fontcuberta i Morral, A.; Xiong, Q.; Arbiol, J. Nano Lett. 2012, 12, 2579-2586.

(31) Mirin, R. P.; Ibbetson, J. P.; Nishi, K.; Gossard, A. C.; Bowers, J. E. Appl. Phys. Lett. 1995, 67, 3795.

(32) Lee, H.; Lowe-Webb, R.; Yang, W.; Sercel, P. C. Appl. Phys. Lett. 1998, 72, 812. 
(33) Bergbauer, W.; Strassburg, M.; Kolper, C.; Linder, N.; Roder, C.; Lahnemann, J.; Trampert, A.; Fundling, S.; Li, S. F.; Wehmann, H. H.; Waag, A. Nanotechnology 2010, 21, 305201 .

(34) Urban, A.; Malindretos, J.; Klein-Wiele, J. H.; Simon, P.; Rizzi, A. New J. Phys. 2014, 16, 019501.

(35) Utama, M. I. B.; Peng, Z.; Chen, R.; Peng, B.; Xu, X.; Dong, Y.; Wong, L. M.; Wang, S.; Sun, H.; Xiong, Q. Nano Lett. 2011, 11, 3051-3057.

(36) Chen, X. J.; Perillat-Merceroz, G.; Sam-Giao, D.; Durand, C.; Eymery, J. Appl. Phys. Lett. 2010, $97,151909$.

(37) Jindal, V.; Shahedipour-Sandvik, F. J. Appl. Phys. 2009, 106, 083115.

(38) Sun, Q.; Yerino, C. D.; Leung, B.; Han, J.; Coltrin, M. E. J. Appl. Phys. 2011, 110, 053517.

(39) Du, D.; Srolovitz, D. J.; Coltrin, M. E.; Mitchell, C. C. Phys. Rev. Lett. 2005, 95, 155503.

(40) Kresse, G.; Hafner, J. Phys. Rev. B 1993, 47, 558-561.

(41) Kresse, G.; Furthmuller, J. Phys. Rev. B 1996, 54, 11169-11186.

(42) Smith, A. R.; Feenstra, R. M.; Greve, D. W.; Neugebauer, J.; Northrup, J. E. Phys. Rev. Lett. 1997, 79, 3934.

(43) Zywietz, T.; Neugebauer, J.; Scheffler, M. Appl. Phys. Lett. 1998, 73, 487.

(44) Henkelman, G.; Jónsson, H. J. Chem. Phys. 2000, 113, 9978. 
(45) Henkelman, G.; Uberuaga, B. P.; Jónsson, H. J. Chem. Phys. 2000, 113, 9901.

(46) Bietti, S.; Somaschini, C.; Esposito, L.; Fedorov, A.; Sanguinetti, S. J. Appl. Phys. 2014, 116, 114311.

(47) Wang, X.; Che, S. B.; Ishitani, Y.; Yoshikawa, A. J. Appl. Phys. 2006, 99, 073512.

(48) Consonni, V.; Hanke, M.; Knelangen, M.; Geelhaar, L.; Trampert, A.; Riechert, H. Phys. Rev. B 2011, 83, 035310.

(49) Yu, X.; Wang, H.; Lu, J.; Zhao, J.; Misuraca, J.; Xiong, P.; von Molnár, S. Nano let. 2012, 12, 5436-5442. 


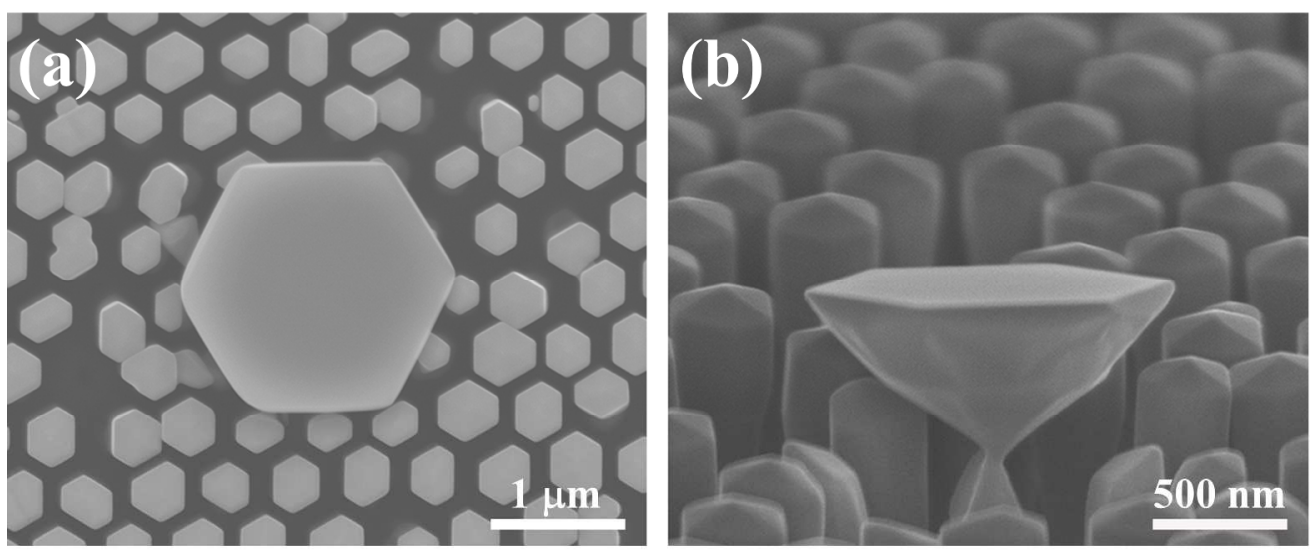

Figure 1. (a) Top-view and (b) tilt-view SEM images of the inverted pyramid that emerged in In-polarity InN NWs. $238 \times 98 \mathrm{~mm}(300 \times 300 \mathrm{DPI})$

23
24
25
26
27
28
29
30
31
32
33
34
35
36
37
38
39
40
41
42
43
44
45
46
47
48
49
50
51
52
53
54
55
56
57
58
59
60



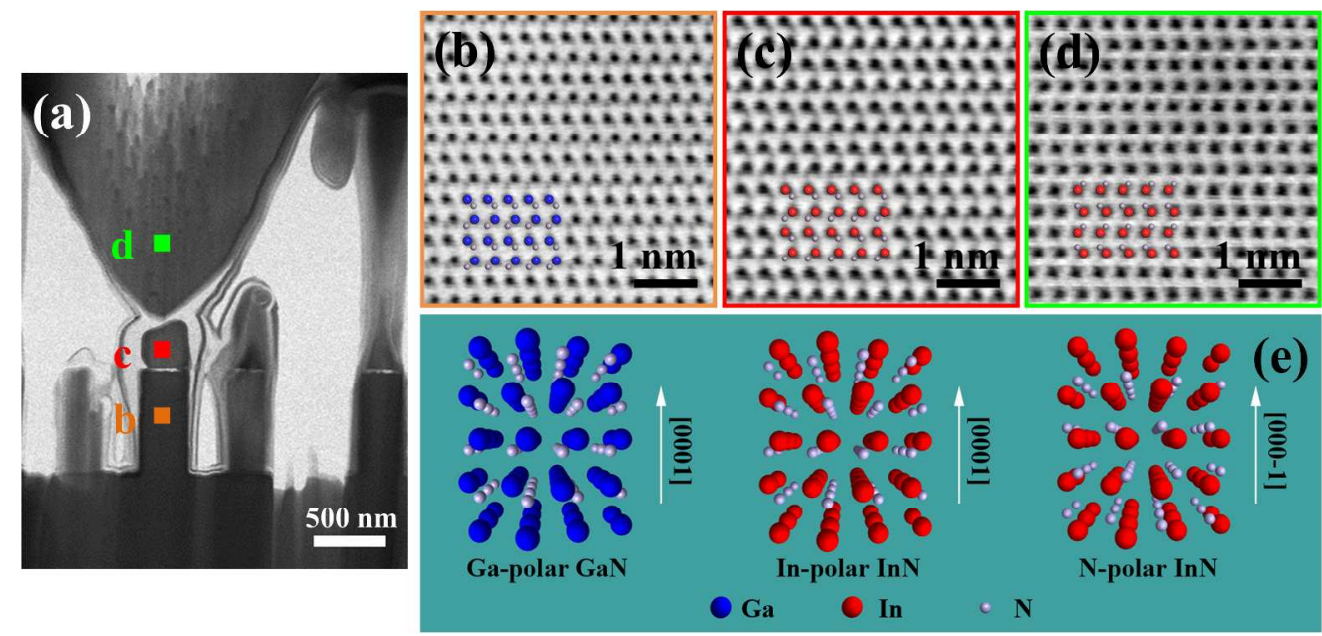

Figure 2. (a) TEM image of an inverted InN NW. (b), (c), and (d) are ABF-STEM images of the zones marked as brown square (GaN pillar), red square (InN wire), and green square (inverted pyramid) in (a), respectively. Ga (blue balls), In (red balls), and N (gray balls) atoms were embedded in the ABF-STEM images to show the atomic stack order. (e) Atomic schematics of Ga-polarity GaN, In- and N-polarity InN. $249 \times 119 \mathrm{~mm}(300 \times 300$ DPI) 


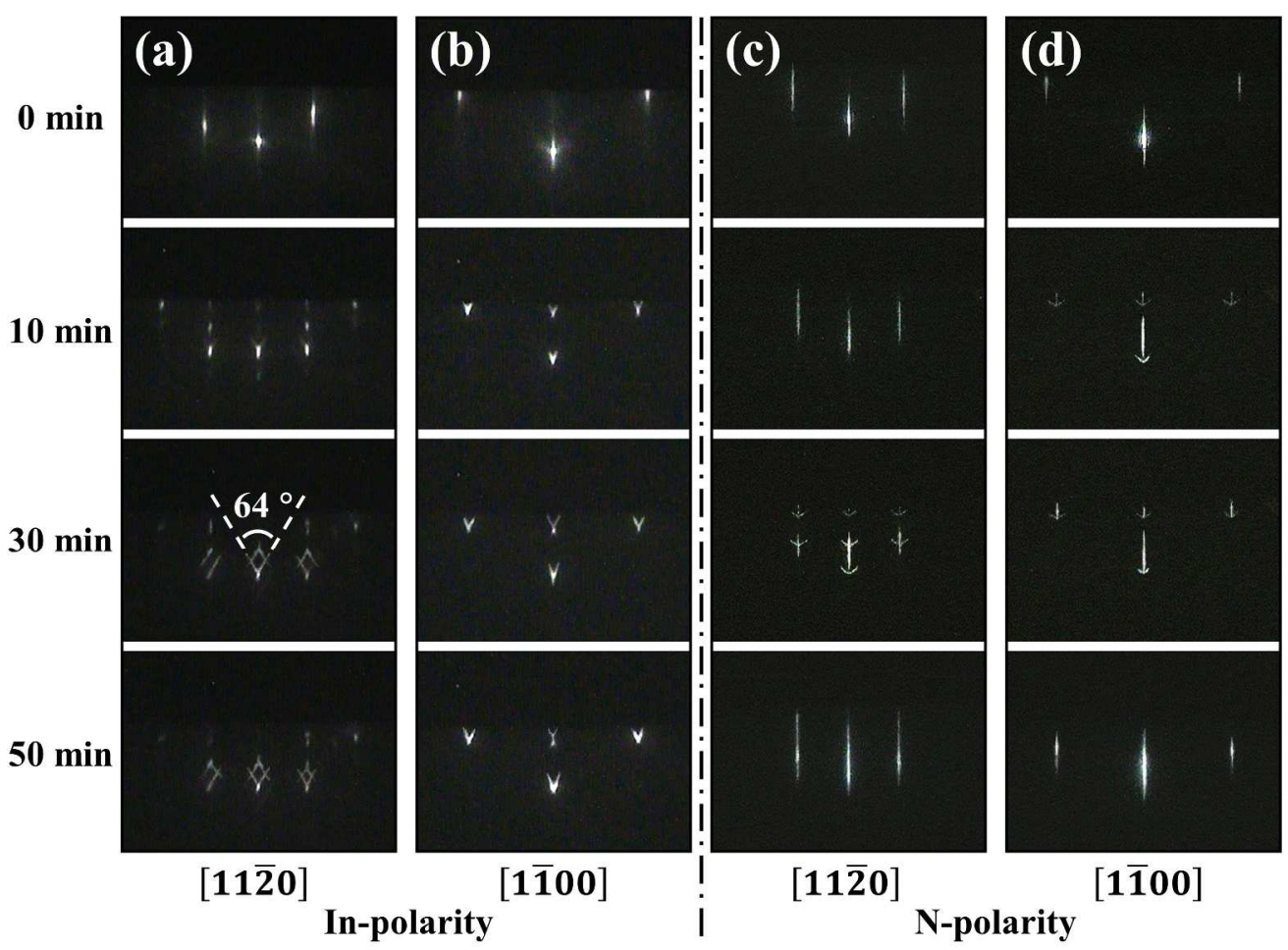

Figure 3. Evolution of RHEED patterns of $(a, b)$ In- and (c, d) N-polarity InN NWs along the [1120] and [1100] azimuths with growth time of $\mathrm{t}=0,5,30$, and $60 \mathrm{~min}$. The white dashed line in (a) shows an included angle of $64^{\circ}$ that indicates a diffraction pattern from the $\operatorname{InN}\left\{11^{\top} 03\right\}$ family. $247 \times 180 \mathrm{~mm}(300 \times 300 \mathrm{DPI})$ 

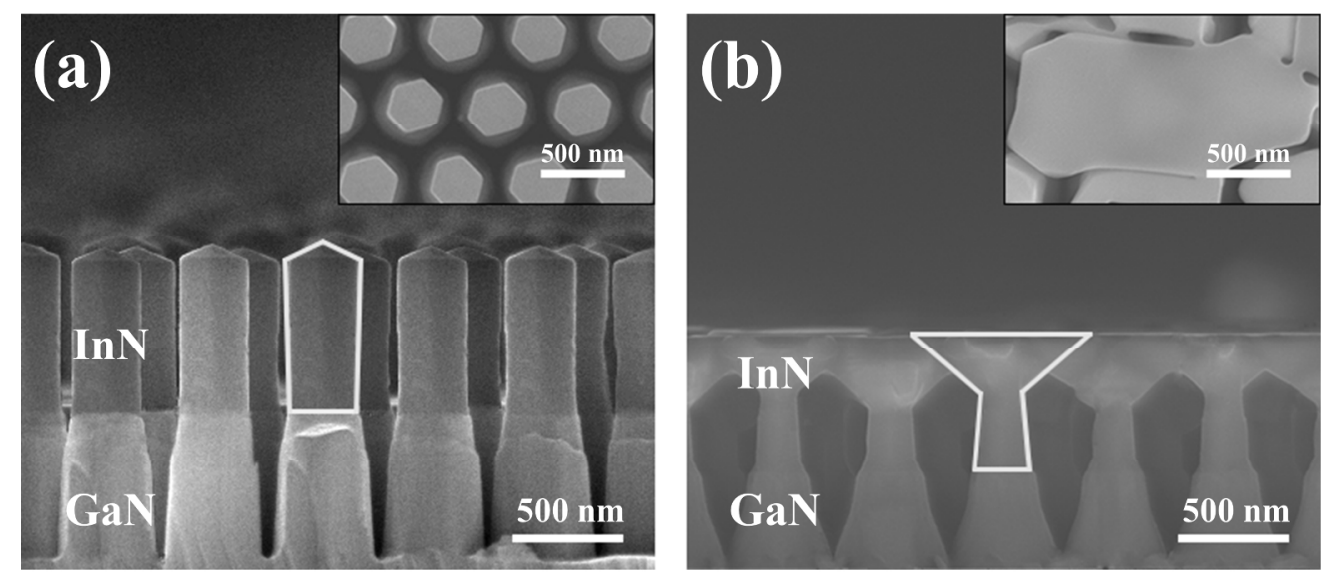

Figure 4. Cross-section SEM images of (a) In-polarity and (b) N-polarity InN NWs. Insets are top-views of the corresponding nanowires. The gray frames show the outline of opposite polarities InN NWs with pyramid-shaped growth fronts and inverted growth fronts, respectively. $238 \times 102 \mathrm{~mm}(300 \times 300 \mathrm{DPI})$ 

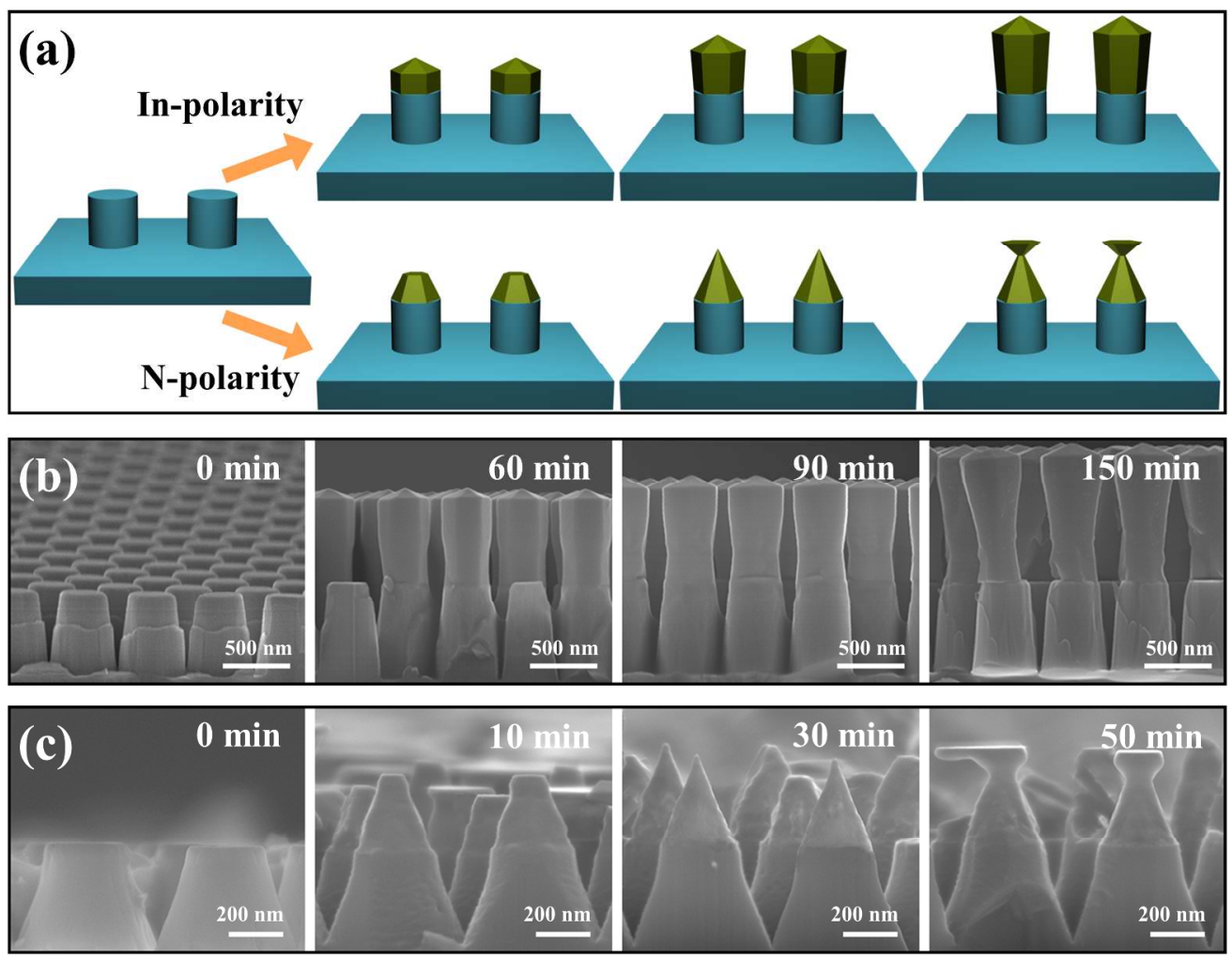

Figure 5. (a) A schematic model of different growth stages of InN NWs with opposite polarities. Tilt-view SEM images of (b) In- and (c) N-polarity InN NWs with growth time. $240 \times 186 \mathrm{~mm}(300 \times 300 \mathrm{DPI})$ 
1

2

3

4

5

6

7

8

9

10

11

12

13

14

15

16

17

18

19

20

21

22

23

24

25

26

27

28

29

30

31

32

33

34

35

36

37

38

39

40

41

42

43

44

45

46

47

48

49

50

51

52

53

54

55

56

57

58

59

60
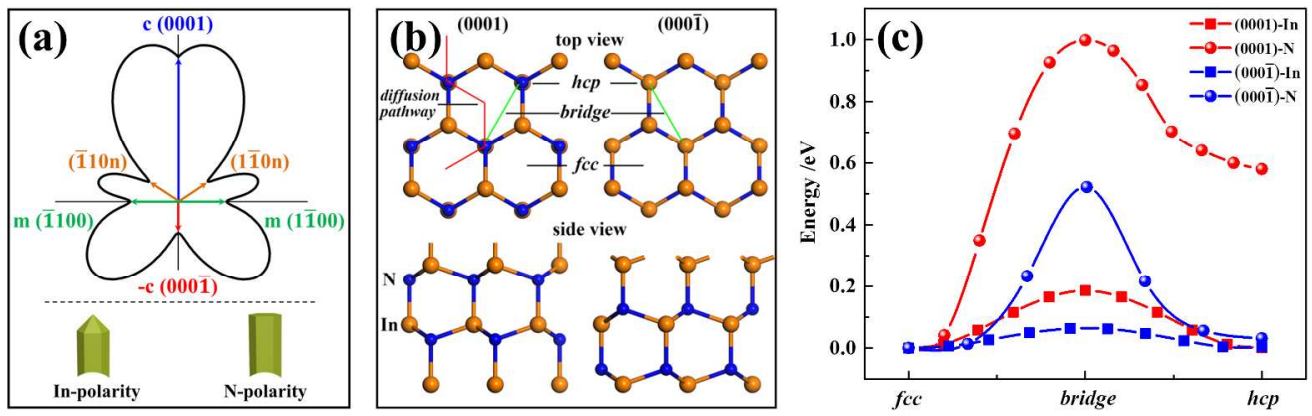

Figure 6. (a) The kinetic Wulff's plots for InN across the (112 0 ) plane: the blue, brown, green, and red arrows correspond to the (0001), $\{11 \overline{0} \mathrm{n}\},\{11 \overline{00}\}$, and $\left(0001^{-}\right)$planes, and solid line shows the extrapolated velocity values. (b) Atomic structures (top view and side view) for (0001) and (0001 ${ }^{-}$) In terminated surfaces. The red line at the top left marks the diffusion pathway corresponding to (c). Three key adsorption sites, fcc, bridge, and hcp, are also marked in this figure. (c) Total energy for In and $\mathrm{N}$ adatoms on $(0001)$ and $\left(0001^{-}\right)$surfaces, which was calculated based on the atomic structure shown in (b). Zero energy corresponds to the energetically lowest adsorption site. $252 \times 78 \mathrm{~mm}(300 \times 300 \mathrm{DPI})$ 

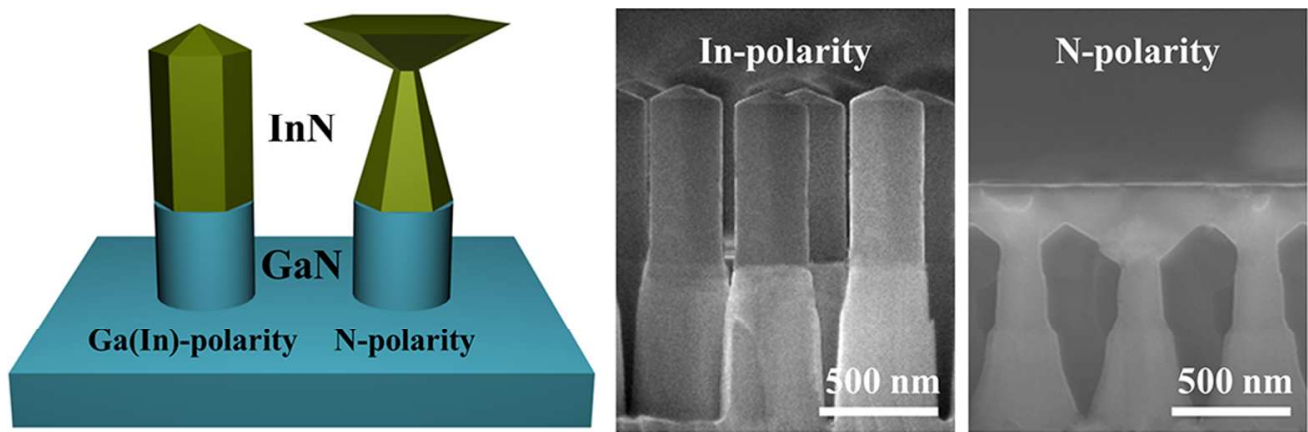

$90 \times 29 \mathrm{~mm}(300 \times 300$ DPI $)$ 